\title{
Deletion of chromosome 13 in Moebius syndrome
}

\author{
J J Slee, R D Smart, D L Viljoen
}

\begin{abstract}
A girl aged 21/2 years with Moebius syndrome was found to have a deletion of band q12.2 in chromosome $13(46, X X, \operatorname{del}(13)(q 12.2))$. This is the second report concerning involvement of chromosome $13 \mathbf{q}$ and Moebius syndrome. The observation raises the possibility that a gene responsible for Moebius syndrome is located in this region of chromosome 13.
\end{abstract}

Moebius syndrome (MS) consists of congenital facial nerve paralysis with or without paralysis of other cranial nerves (predominantly abducens), and may be associated with congenital abnormalities of the extremities and orofacial structures. The cranial nerve anomalies may be complete, partial, unilateral, or bilateral. 12

In this paper we present a child aged $21 / 2$ years with MS and a deletion of chromosome 13 . To the best of our knowledge this is only the second time that the association of MS and a chromosome anomaly in the region of $13 \mathrm{q}$ has been documented. ${ }^{3}$

\section{Case report}

The proband, a female aged $2 \frac{1}{2}$ years, was the only child of non-consanguineous parents. Her birth weight at term was $3150 \mathrm{~g}$ after an uncomplicated pregnancy and delivery. No exposure to known teratogenic agents occurred during the pregnancy.

She was noted at birth to have left facial palsy and a small tongue. She suckled poorly on the breast in the early neonatal period, but was subsequently satisfactorily bottle fed. There was no history of similarly affected persons in the family. At the time of birth, maternal and paternal ages were 36 and 45 years, respectively.

At the age of 3 months her weight and height were normal. She had mask-like facies, a flattened nasal

Department of Human Genetics and MRC Unit for Skeletal Dysplasias, University of Cape Town Medical School, Observatory 7925, Cape Town, South Africa. J J Slee, R D Smart, D L Viljoen

Correspondence to Dr Slee.

Received for publication 1 October 1990.

Revised version accepted for publication 22 October 1990. bridge, hypertelorism, synophrys, bilateral partial abducens nerve palsies, and left facial nerve palsy. In addition, micrognathia, a high arched palate, and a small tongue with hypoglossal weakness were apparent. She had no congenital abnormalities of the extremities. The child has been reappraised on several occasions and the physical features have persisted (fig 1).

\section{CYTOGENETIC STUDIES}

Chromosome preparations were obtained from 72 hour lymphocyte cultures. Prometaphase plates were produced using a modified ethidium bromide technique, as described by Ikeuchi. ${ }^{4}$ GTG banding showed a deletion of band q12.2 in chromosome 13 (fig 2). All the acrocentric chromosomes showed positive NOR staining. The centromeric area of the normal chromosome 13 was large, a feature which was confirmed by $\mathrm{C}$ banding, but this was not considered to be abnormal. The chromosome constitution was 46,XX,del(13)(q12.2). The mother's karyotype appeared normal, but paternal chromosomal studies could not be undertaken as the father had died.

It seems likely that the normal chromosome 13 with

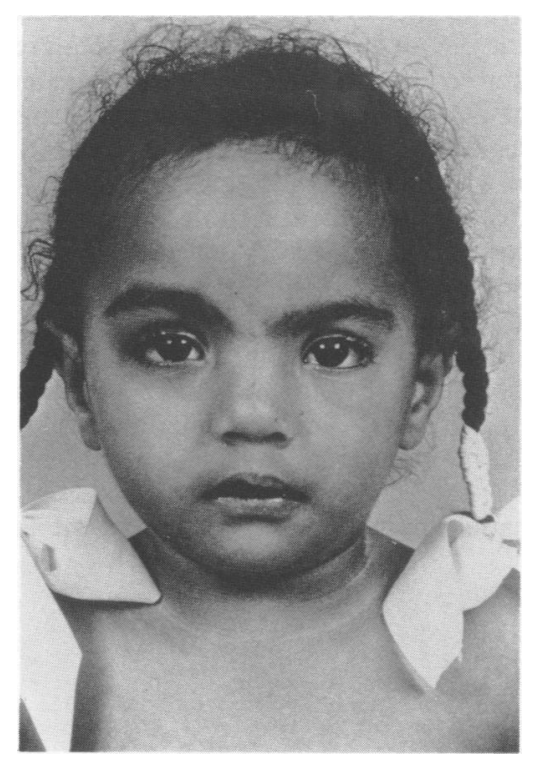

Figure 1 The patient aged $21 / 2$ years. 


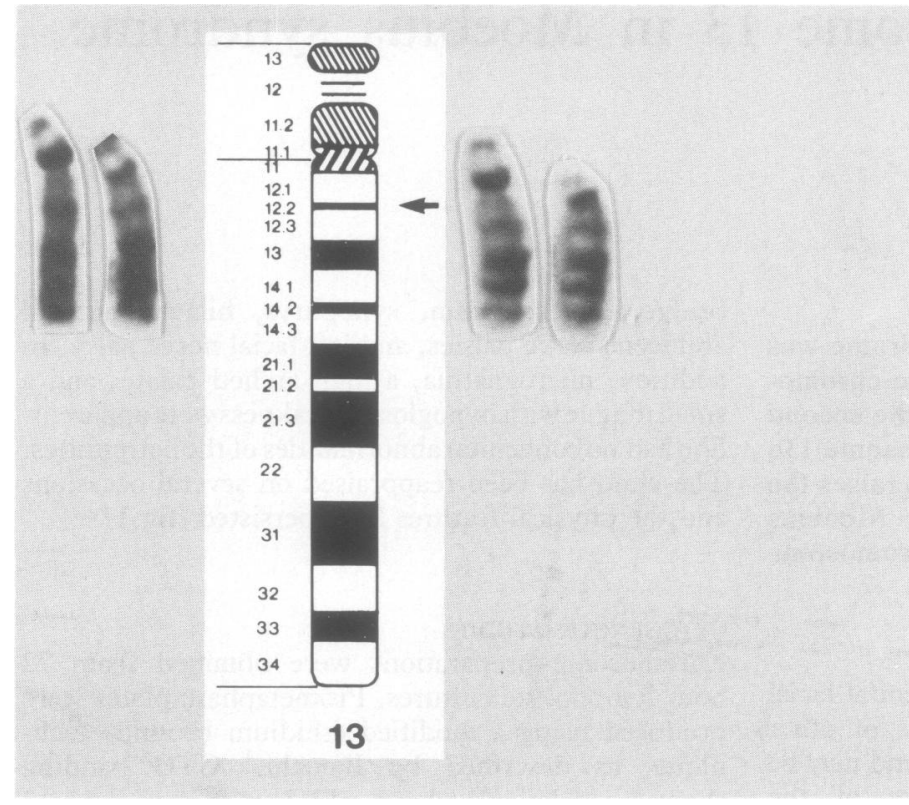

Figure 2 GTG banding of chromosomes 13. The deleted chromosome is on the right. The missing band is arrowed.

the large centromeric variant was inherited from the father as it is not present in the mother's chromosomes. Therefore, the chromosome carrying the de novo deletion is maternally derived.

\section{Discussion}

The affected child showed classical features of Moebius syndrome in association with a deletion of chromosome 13 (46,XX,del(13)(q12.2). In a previous report Ziter et $\mathrm{al}^{3}$ described a three generation pedigree of a Moebius syndrome variant (congenital facial diplegia, finger flexion deformities, and mild intellectual impairment) cosegregating with an apparent balanced reciprocal translocation between chromosomes 1 and $13(46, \mathrm{XY}, \mathrm{t}(1 ; 13)(1 \mathrm{qter} \rightarrow 1 \mathrm{p} 34::$ $13 q 13 \rightarrow 13 q$ ter;13q13 $\rightarrow 13$ pter:: lpter $\rightarrow 1$ p34)).

Although the transmission appeared balanced, Ziter $e t$ al suggested the possibility that a microdeletion could be present at the breakpoint, resulting in the abnormal phenotype. Such a deletion could have occurred at the breakpoint in chromosomes 13 (q13) or 1 (p34).

In our patient no translocation was present and the loss of chromosomal material had occurred at band q12.2. This then is the second case where the same region of chromosome 13 has been implicated in MS. Although considerable evidence of heterogeneity in MS has accumulated, ${ }^{5}$ we propose that a gene for MS is located on, or near, band q12.2 on chromosome 13 . It would be appropriate to undertake further studies in this region, using molecular techniques, in order to elucidate this problem.

This project was supported by grants from the South African Medical Research Council, the Mauerberger Foundation, the Harry Crossley Fund, and the University of Cape Town Staff Research Fund.

1 Jones KL. Smith's recognizable patterns of human malformations. 4th ed. Philadelphia: Saunders, 1988.

2 Kumar D. Moebius syndrome. F Med Genet 1990;27:122-6.

3 Ziter FA, Wiser WC, Robinson A. Three-generation pedigree of a Mobius syndrome variant with chromosomal translocation. Arch Neurol 1977;34:437-42.

4 Ikeuchi T. Inhibitory effect of ethidium bromide on mitotic chromosome condensation and its application to high-resolution chromosome banding. Cytogenet Cell Genet 1984;38:56-61.

5 McKusick VA. Mendelian inheritance in man. 8th ed. Baltimore: Johns Hopkins University Press, 1988. 